\title{
Bacillus subtilis DE111 intake may improve blood lipids and endothelial function in healthy adults
}

\author{
R.E. Trotter, A.R. Vazquez, D.S. Grubb, K.E. Freedman, L.E. Grabos, S. Jones, C.L. Gentile, C.L. Melby, S.A. Johnson \\ and T.L. Weir" \\ Department of Food Science and Human Nutrition, Colorado State University, 502 W Lake St, Fort Collins, CO 80523- \\ 1571,USA; tiffany.weir@colostate.edu
}

Received: 27 February 2020 / Accepted: 22 June 2020

(c) 2020 Wageningen Academic Publishers

OPEN ACCESS C(1)(2) RESEARCH ARTICLE

\begin{abstract}
Cardiovascular disease (CVD) is the leading cause of death in the US and worldwide. By 2030 it is anticipated that CVD will claim the lives of more than 24 million people. Throughout the last decade, researchers have investigated the role of the gut microbiota in the development of CVD. Evidence exists for a positive correlation between Bifidobacterium and vascular function, glucose tolerance, and reduced systemic inflammation. Another probiotic species, Bacillus subtilis, has also been found to reduce cholesterol levels in human and animal models. In light of these data, we examined various measures of cardiovascular health after consumption of Bifidobacterium animalis subsp. lactis strain BL04, with and without a cocktail of Escherichia coli-targeting bacteriophages (marketed as PreforPro), Bacillus subtilis strain DE111 or a maltodextrin-based placebo in a healthy human population. In a randomised, double-blind, placebo-controlled 4-week intervention conducted in individuals 18 to 65 years of age with a body mass index of 20 to 34.9, we saw no significant changes in measured CVD parameters among individuals consuming B. lactis with or without bacteriophages. However, B. subtilis supplementation resulted in a significant reduction in total cholesterol relative to baseline measures $(-8 \mathrm{mg} / \mathrm{dl} ; P=0.04$, confidence interval (CI): -13.40, -0.19$)$, as well as non-high-density lipoprotein-cholesterol ( $-11 \mathrm{mg} / \mathrm{dl} ; P=0.01, \mathrm{CI}:-12.43,-2.07)$. In addition we observed trending improvements in endothelial function $(P=0.05, C I$ : $-0.003,0.370)$ and in low-density lipoprotein-cholesterol $(P=0.06, C I:-12.29,0.2864)$. Strikingly, these effects were seen in a largely healthy population. These data suggest that $B$. subtilis supplementation may be beneficial for improving risk factors associated with CVD. Further studies in populations of older adults or those with dyslipidaemia and endothelial dysfunction is warranted.
\end{abstract}

Keywords: bacteriophage, Bifidobacterium lactis, Bacillus subtilis, cardiovascular disease, vascular function, probiotic

\section{Introduction}

Emerging evidence supports a role for dysbiosis, or imbalance, of the gut microbiota in the development of cardiovascular disease (CVD) (Battson et al., 2018b; Cani et al., 2008; Koren et al., 2011; Kumar et al., 2012; Xie et al., 2011; Ziganshina et al., 2016). As a result, therapies to restore balance to the gut microbiota are being explored as a novel target for CVD prevention and/or treatment. Current therapeutic modalities for altering the gut microbiota include prebiotics, probiotics, faecal microbiota transplantations, and antibiotics (Battson et al., 2018b). Among these, probiotics, which are live beneficial microbes, and prebiotics, or compounds that induce the growth of beneficial microorganisms in the gut, are readily available to consumers and make up a large and growing segment of the dietary supplement market (Gibson et al., 2017; Hill et al., 2014).

Several probiotic and prebiotic supplements have shown promise for attenuating various cardiovascular risk factors, including indices of endothelial dysfunction and dyslipidaemia. Lactobacillus plantarum strain Lp299V supplementation improved brachial flow-mediated dilation in men with stable coronary artery disease (Malik et al., 2018) and a high dose, multi-species probiotic taken by 
postmenopausal women for 12 weeks was associated with improvements in several measures of vascular function, including pulse wave velocity and pulse wave analysis (Szulińska et al., 2018). We have previously shown decreased populations of Bifidobacterium are correlated with obesity-associated vascular dysfunction in animal models (Battson et al., 2018a). In addition, Bifidobacterium lactis supplementation has been shown to improve endothelial function in human populations. In a healthy Japanese cohort, yogurt containing B. lactis was consumed for 12 weeks in combination with arginine and resulted in a significant improvement in reactive hyperaemia index (RHI) scores, a validated measure of endothelial function in humans (Johnson et al., 2019), and systolic and diastolic blood pressure (Matsumoto et al., 2019). A separate study found that 45-day supplementation of fermented milk containing $B$. lactis in individuals with metabolic syndrome significantly reduced total and low-density lipoproteincholesterol (LDL-c) (Bernini et al., 2016).

There is the possibility that the combination supplemental intake of a bacteriophage cocktail could enhance probiotic supplementation. We previously showed that 4 week consumption of the commercial phage cocktail, PreforPro (Deerland Enzymes, Kennesaw, GA, USA), increased Bifidobacterium populations and reduced specific proinflammatory bacteria, such as Clostridium perfringens and Escherichia coli (Febvre et al., 2019). These phages are marketed as prebiotics, although they do not meet the current definition of prebiotic (Gibson et al., 2017) and differ from traditional fibre-based prebiotics in that they infect specific bacteria ( $E$. coli, in this case), potentially allowing beneficial bacteria to thrive due to reduced resource competition and increased fuel sources from released contents of lysed bacteria. Because bacteriophages exhibit a high degree of host specificity, they do not cause global perturbations to the gut microbiota, such as those seen with antibiotic usage (Febvre et al., 2019).

Another probiotic, Bacillus subtilis, has also shown promise in attenuating glucose intolerance and dyslipidaemia. These bacteria form temperature and acid-resistant spores, which provide $B$. subtilis with a longer shelf life and increased ability to survive the digestive tract relative to other probiotics. Bacillus subtilis is naturally found in several soy-based fermented foods from Asia and Africa, such as natto (Japan), cheonggukjang (Korea), and soy-daddawa (Nigeria). In addition, when metabolically active, B. subtilis produces a variety of exopolysaccharides (EPS), which have gained attention for their potential therapeutic benefits. In a previous study, diabetic rats were supplemented with B. subtilis EPS for 4 weeks, resulting in reduced blood glucose concentrations, total cholesterol, LDL-c, very lowdensity lipoprotein-cholesterol (VLDL-c) and triglycerides and elevated high density lipoprotein cholesterol (HDL-c) (Ghoneim et al., 2016). Another study demonstrated that
B. subtilis SPB1 biosurfactant supplementation in diabetic rats produced anti-lipidemic and anti-diabetic properties (Zouari et al., 2015). Thus, B. subtilis is an ideal candidate to explore for potential cardiovascular benefits in humans.

In light of mounting evidence that the microbiota can influence development and progression of CVD, the current study sought to explore whether probiotic/ prebiotic consumption would improve various measures of cardiovascular health in a human population. We conducted a randomised, double-blind, four-arm, placebo-controlled clinical trial in healthy adults that tested whether four-week consumption of (1) maltodextrin placebo; (2) B. animalis subsp. lactis; herein B. lactis) alone, or (3) in combination with a cocktail of $E$. coli-targeting bacteriophages; and (4) B. subtilis, altered risk factors of CVD. The primary outcome measures included blood pressure and pulse wave analysis measured via SphygmoCor, endothelial function (determined by EndoPat), and plasma lipid levels. We hypothesised that probiotic consumption would improve one or more measures of cardiovascular function in our participant population, and that simultaneous supplementation with $E$. coli-targeting bacteriophages might further enhance these beneficial cardiovascular effects.

\section{Materials and methods}

\section{Participant characteristics}

94 healthy male and female adults aged 18-65 years with a body mass index (BMI) of $20-34.9 \mathrm{~kg} / \mathrm{m}^{2}$ were recruited into the study (Figure 1). Participants were recruited from the Fort Collins (CO, USA) area by email, social media platforms, fliers, and word of mouth. Participants were prescreened for eligibility through a phone interview. Those who met the inclusion criteria were scheduled for an onsite screening visit to confirm inclusion/exclusion criteria, which are summarised in Table 1. Qualified participants were asked to maintain their regular exercise and dietary habits throughout the study, abstain from supplemental prebiotics or probiotics, and to limit alcohol consumption to one to two drinks per day or no more than seven drinks per week. Of the total enrolled participants $(n=94), 88$ completed the study. Baseline characteristics for participants included in this study are presented in Table 2.

\section{Study design}

The study was conducted as a randomised, double-blind, placebo-controlled, four-arm clinical trial. Once written informed consent was secured, eligibility was confirmed through assessment of health history, and anthropometric measures of height and weight to determine BMI. After eligibility was confirmed, participants were randomly assigned to one of four coded treatments: (1) maltodextrin 


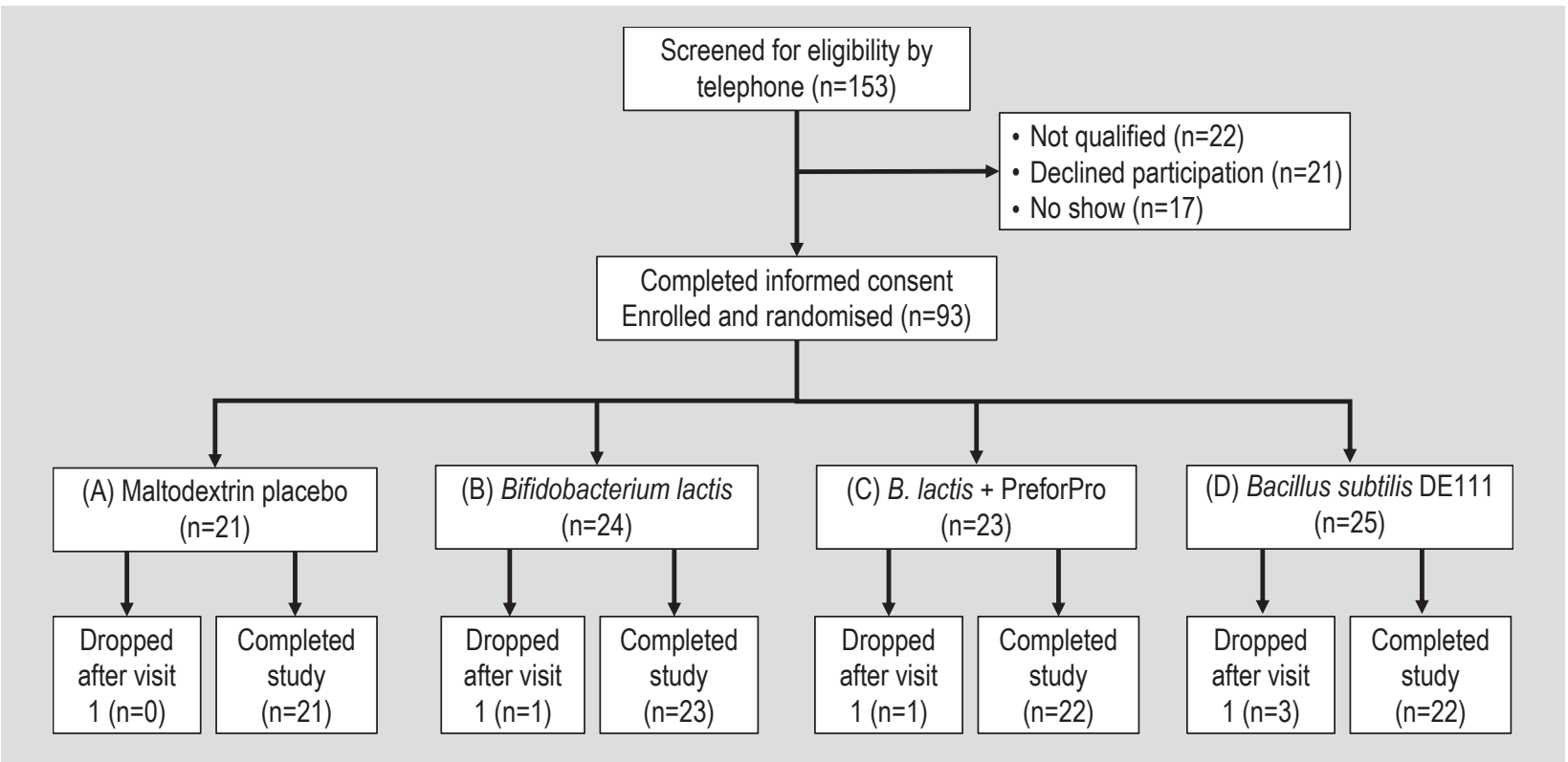

Figure 1. Consort flow diagram of participants through study.

Table 1. Study inclusion and exclusion criteria. ${ }^{1}$

\begin{tabular}{|c|c|}
\hline Inclusion criteria & Exclusion criteria \\
\hline Men and women & Pregnant and breastfeeding women \\
\hline Aged $18-65$ years & $\begin{array}{l}\text { Taking medication that would influence the } \\
\text { endpoints of the study (statins, metformin, } \\
\text { NSAIDS, MAO inhibitors, blood pressure } \\
\text { medications) and taking probiotics and/ } \\
\text { or botanical supplements that target the } \\
\text { gastrointestinal tract or gut microbiota }\end{array}$ \\
\hline \multirow[t]{3}{*}{$\begin{array}{l}\text { Normal, overweight, } \\
\text { or class } 1 \text { obese } \\
\left(\mathrm{BMI} 20-34.9 \mathrm{~kg} / \mathrm{m}^{2}\right)\end{array}$} & $\begin{array}{l}\text { Current diagnosis of cancer, liver or kidney } \\
\text { disease, gastrointestinal diseases, and } \\
\text { metabolic disorders }\end{array}$ \\
\hline & $\begin{array}{l}\text { Antibiotic use within the } 2 \text { months prior to } \\
\text { enrolment }\end{array}$ \\
\hline & $\begin{array}{l}\text { Enrolled in other research studies that might } \\
\text { impact compliance or confound results of the } \\
\text { current study }\end{array}$ \\
\hline
\end{tabular}

placebo ( $\mathrm{n}=21)$; (2) $1 \times 10^{9} \mathrm{cfu}$ Bifidobacterium lactis strain BL04 ( $\mathrm{n}=23)$; 3) $1 \times 10^{6}$ pfu PreforPro bacteriophages + $1 \times 10^{9} \mathrm{cfu}$ B. lactis ( $\mathrm{n}=22$; Deerland Enzymes); or (4) $1 \times 10^{9} \mathrm{cfu}$ Bacillus subtilis strain DE111 ( $\mathrm{n}=22$; Deerland Enzymes). Over the course of the four-week intervention, participants were asked to consume one $15 \mathrm{mg}$ capsule per day of their assigned treatment. The study was conducted at the Colorado State University, Food and Nutrition Clinical Research Laboratory (FNCRL). The study protocol was approved by the Colorado State University Institutional Review Board for Human Subjects (Protocol \#19-9145H).

For experimental clinical sessions, participants were asked to fast for $12 \mathrm{~h}$ prior to arrival in the clinic (including no caffeine, soda, tea, etc.), to abstain from exercise within $12 \mathrm{~h}$ prior to their visit, and to delay consumption of any medication or dietary supplements for $24 \mathrm{~h}$ prior until conclusion of the study visit. Participants were asked to visit the FNCRL at visit one (Day 0) to undergo sample collection (blood) and assessment procedures (weight/height, blood pressure, pulse wave analysis, endothelial function, and medical health history questionnaire). At the end of the four-week treatment period, these same assessments were conducted and blood samples were collected. To aid in determining treatment compliance, participants were also asked to return any unused treatment capsules. Participants were asked to maintain their typical dietary habits over the course of the study to reduce confounding impacts related to dietary changes. Dietary compliance was measured via computer software, automated self-administered 24-h dietary assessment tool (ASA24) (National Institutes of Health, National Cancer Institute, USA) using two 24-h dietary recalls (one week day and one weekend day) collected prior to each clinic visit.

\section{Blood pressure and vascular function}

Participants were asked to lay in a supine position and rest for $10 \mathrm{~min}$. Blood pressure and pulse wave analysis were then assessed (SphygmoCor XCEL; AtCor Medical, Naperville, IL, USA). Each measurement was made three times, and data analysed represent the mean of the three measurements. As previously described (Litwin et 
Table 2. Participant baseline characteristics.

\begin{tabular}{lcccccc} 
Treatment & Male & Female & Height $(\mathbf{c m})$ & Weight $(\mathbf{k g})$ & BMI $^{1}$ & Age \\
& & & & & & \\
Placebo & 8 & 13 & $169.9 \pm 8.2$ & $71.3 \pm 10.6$ & $24.7 \pm 2.8$ & $36.5 \pm 13.0$ \\
Bifidobacterium lactis BL04 & 10 & 13 & $169.9 \pm 8.2$ & $71.5 \pm 10.7$ & $24.7 \pm 2.8$ & $36.4 \pm 13.2$ \\
B. lactis BL04 + PreforPro & 7 & 15 & $170.1 \pm 8.2$ & $71.6 \pm 10.4$ & $24.7 \pm 2.7$ & $36.1 \pm 13.3$ \\
Bacillus subtilis DE111 & 10 & 12 & $169.8 \pm 8.4$ & $71.6 \pm 10.7$ & $24.7 \pm 2.8$ & $36.6 \pm 13.2$ \\
\hline BMI = body mass index. & & & & & &
\end{tabular}

al., 2019), brachial pulse pressure was calculated as the difference between mean systolic blood pressure and diastolic blood pressure. Central aortic blood pressure and related hemodynamic parameters (e.g. aortic mean arterial pressure, aortic pulse pressure) were derived from brachial pressure waveforms using a validated transfer function and automatically recorded. Aortic augmentation index (AIx), and AIx@75 (normalised to 75 beats per min), which are reflective of arterial stiffness and vascular tone (Kelly et al., 2001) were automatically calculated as the ratio between augmented aortic pressure (i.e. difference between the first and second derived aortic systolic peaks) and aortic pulse pressure. Endothelial function was measured via peripheral arterial tonometry (EndoPAT 2000; Itamar Medical Ltd, Hefa, Israel). In order to maintain consistency and quality in data collection the following procedure was performed in accordance with requirements specified by the manufacturer as previously described (Litwin et al., 2019). Participants are classified as having endothelial dysfunction if the RHI-score $\leq 1.67$.

\section{Plasma lipids}

Blood samples $(100 \mathrm{ml})$ were collected from the antecubital vein in a lithium heparin tube and immediately analysed for total cholesterol (TC), HDL-c, triglycerides (TRIGs), non-high-density lipoprotein cholesterol (non-HDL-c), total cholesterol/HDL ratio (TC/HDL), LDL-c and VLDL-c using a Lipid Panel Reagent Disc on the Piccolo Xpress Chemistry Blood Analyzer (Abaxis, Union City, CA, USA).

\section{Statistical analysis}

R software, version 3.6.1 (2019-07-05), was used for the following statistical analyses. Data were evaluated for assumption of normality and missing values were excluded from the analysis. To determine if there were differences among baseline (Pre) participant characteristics, we compared the treatment groups using one-way ANOVA. Baseline to post-treatment comparisons within groups was assessed using two-tailed, paired Student's t-tests. In addition, we used GraphPad Prism, version 8.3.0 (La Jolla, CA, USA), to conduct a one-way ANOVA with Dunnett's
Multiple Comparison post-hoc test to determine baseline normalised differences between the placebo and treatment groups after the 4-week intervention. A $P$-value of $\leq 0.05$ was considered statistically significant and parameters with a $P=0.05-0.08$ were considered statistical trends. A Cohen's $d$ effect size was calculated as a quantitative measure of determining the magnitude of difference between two means (treatment and placebo). The Cohen's $d$ scores were calculated using $d=[(\Delta$ Treatment $-\Delta$ Placebo $) /$ pooled standard deviation] and the standard definitions of small (0.2), moderate (0.5), and large (0.8), and very large (1.2) were applied (Cohen, 2013; Sawilowsky, 2009).

\section{Results}

\section{Compliance and anthropometric measures}

Total study compliance with the treatment regimen was 95\% with individual compliance ranging from $73-100 \%$. All individual data was included in the analysis, regardless of compliance, under the intention-to-treat principle (Yelland et al., 2015). Compliance to specific treatments were as follows: Placebo: average compliance $=97 \%$ (range $=77$ $100 \%$ ); B. lactis BL04: average compliance $=95 \%$ (range $=$ 80-100\%); B. lactis BL04 + PreforPro: average compliance $=95 \%$ (range $=73-100 \%)$; $B$. subtilis DE111: average compliance $=96 \%($ range $=83-100 \%)$.

Overall, there was low compliance for completing the selfreported 24 -h food recalls, with only $~ 53 \%$ of participants providing both baseline and 4-week dietary intake information. Compliance within each of the treatment groups was as follows: Placebo: average compliance $=$ $48 \%$, B. lactis BL04: average compliance $=58 \%$, B. lactis BL04 + PreforPro: average compliance $=57 \%, B$. subtilis DE111: average compliance $=59 \%$. Based on available data, there was notable variability between individuals, but there were no significant changes in dietary intake of total calories, macronutrients, fibre, cholesterol, or saturated fat from baseline to study completion within any of the treatment groups (Table 3). There was a significant difference in grams of saturated fats (SAT) consumed at baseline among treatments, however, pairwise 
Table 3. Self-reported dietary intake.,

\begin{tabular}{|c|c|c|c|c|c|c|c|c|}
\hline & \multicolumn{2}{|c|}{ Placebo $(n=10)$} & \multicolumn{2}{|c|}{ Bifidobacterium lactis BL04 ( $n=14)$} & \multicolumn{2}{|c|}{ PreforPro $+B$. lactis $(n=12)$} & \multicolumn{2}{|c|}{ Bacillus subtilis DE111 $(n=13)$} \\
\hline & Pre & Post & Pre & Post & Pre & Post & Pre & Post \\
\hline Energy (kcal) & $2,269 \pm 999$ & $2,089 \pm 982$ & $1,775 \pm 735$ & $1,853 \pm 675$ & $1,739 \pm 474$ & $1,905 \pm 719$ & $2,238 \pm 741$ & $2,389 \pm 638$ \\
\hline $\mathrm{PRO}(\mathrm{g})$ & $99 \pm 50$ & $96 \pm 50$ & $85 \pm 49$ & $74 \pm 29$ & $70 \pm 18$ & $68 \pm 27$ & $96 \pm 40$ & $102 \pm 48$ \\
\hline TFAT (g) & $103 \pm 56$ & $86 \pm 42$ & $73 \pm 27$ & $84 \pm 44$ & $66 \pm 30$ & $73 \pm 29$ & $95 \pm 40$ & $101 \pm 32$ \\
\hline $\operatorname{SFAT}(\mathrm{g})$ & $33 \pm 18$ & $27 \pm 14$ & $21 \pm 9$ & $29 \pm 21$ & $19 \pm 6$ & $23 \pm 12$ & $32 \pm 19$ & $34 \pm 15$ \\
\hline $\mathrm{CHOL}(\mathrm{mg})$ & $358 \pm 259$ & $324 \pm 271$ & $282 \pm 299$ & $277 \pm 281$ & $215 \pm 101$ & $180 \pm 129$ & $367 \pm 221$ & $439 \pm 308$ \\
\hline CARB $(g)$ & $240 \pm 104$ & $225 \pm 118$ & $194 \pm 103$ & $199 \pm 91$ & $209 \pm 54$ & $222 \pm 71$ & $223 \pm 79$ & $237 \pm 73$ \\
\hline Fibre (g) & $25 \pm 11$ & $22 \pm 12$ & $27 \pm 19$ & $23 \pm 16$ & $26 \pm 21$ & $24 \pm 14$ & $21 \pm 6$ & $27 \pm 10$ \\
\hline
\end{tabular}

comparisons between treatment groups and Placebo showed only a trending difference $(P=0.055)$ between the Placebo and B. lactis BL04 + PreforPro, with no other pairwise differences. Furthermore, there were no differences between any treatment and the Placebo with regard to change in intake over the course of the study. Finally, neither diet nor treatment influenced body weight over the course of the study; the average BMI was $\sim 24 \mathrm{~kg} / \mathrm{m}^{2}$ (Table 2) for all groups both pre- and post-treatment and average weights fluctuated by $<1 \mathrm{~kg}$ in each treatment group.

\section{Blood pressure and vascular function}

A meta-analysis of human clinical trials recently demonstrated that probiotics have a modest modulatory effect on systolic and diastolic blood pressure; therefore, we included this as an outcome in our study (Khalesi et al., 2014). We found no significant baseline differences in systolic or diastolic blood pressure between groups (Table 4). Furthermore, although there was a small number of individuals within each group that could be classified as having elevated blood pressure $(120-129 /<80)$ or hypertensive (stage 1 or 2$)(\geq 130 / \geq 80)$, the group averages fell within a clinically healthy range. There were no significant changes within groups from baseline to post-treatment (Table 4). Likewise, the average change in blood pressure from baseline to post-treatment was not significantly different when comparing treatments to the Placebo.

Table 5 shows the additional cardiovascular outcomes measured including mean arterial pressure, aortic pulse pressure, heart rate, aortic pressure, and both nonnormalised (AIx) and heart-rate normalised augmentation index (AIx@75). The baseline group values were not significantly different and there were no significant group differences from pre- to post-intervention (Table 5).

Using RHI scores generated by the EndoPAT technology as a measure of endothelial function, we found no significant baseline differences across treatment groups (Table 6). Likewise, the response from baseline (pre to post comparison) in Placebo, B. lactis and PreforPro + $B$. lactis treatment groups was not statistically significant. However, within the B. subtilis DE111 treatment group, there was a trending improvement in RHI from baseline to post-intervention (Figure $2 \mathrm{~A} ; P=0.05$; $\mathrm{CI}$ - $-0.003,0.370$ ). However, using a Cohen's $d$ score, it was determined that the magnitude of this effect relative to the placebo was small (Cohen's $d=0.19$ ).

Table 4. Systolic and diastolic blood pressure across time and treatment groups. ${ }^{1}$

\begin{tabular}{|c|c|c|c|c|c|c|c|c|}
\hline & \multicolumn{2}{|c|}{ Placebo ( $n=22)$} & \multicolumn{2}{|c|}{ Bifidobacterium lactis BL04 ( $\mathrm{n=23)}$} & \multicolumn{2}{|c|}{ PreforPro + B. lactis $(n=22)$} & \multicolumn{2}{|c|}{ Bacillus subtilis DE111 ( $\mathrm{n}=22)$} \\
\hline & Pre & Post & Pre & Post & Pre & Post & Pre & Post \\
\hline Systolic BP (mmHg) & $117 \pm 12$ & $116 \pm 11$ & $119 \pm 12$ & $117 \pm 10$ & $119 \pm 12$ & $117 \pm 10$ & $118 \pm 12$ & $117 \pm 10$ \\
\hline Diastolic BP (mmHg) & $70 \pm 9$ & $70 \pm 8$ & $71 \pm 9$ & $71 \pm 8$ & $71 \pm 9$ & $71 \pm 8$ & $71 \pm 9$ & $71 \pm 8$ \\
\hline
\end{tabular}


Table 5. Pulse wave analysis across time and treatment groups.

\begin{tabular}{|c|c|c|c|c|c|c|c|c|}
\hline & \multicolumn{2}{|c|}{ Placebo $(n=22)$} & \multicolumn{2}{|c|}{$\begin{array}{l}\text { Bifidobacterium lactis } \\
\text { BL04 }(n=23)\end{array}$} & \multicolumn{2}{|c|}{ PreforPro + B. lactis $(n=22)$} & \multicolumn{2}{|c|}{$\begin{array}{l}\text { Bacillus subtilis } \\
\text { DE111 }(n=22)\end{array}$} \\
\hline & Pre & Post & Pre & Post & Pre & Post & Pre & Post \\
\hline Aortic MAP (mmHg) & $84.08 \pm 9.22$ & $84.27 \pm 8.81$ & $84.18 \pm 9.18$ & $84.17 \pm 8.72$ & $84.22 \pm 9.12$ & $84.06 \pm 8.71$ & $84.21 \pm 9.16$ & $84.15 \pm 8.73$ \\
\hline Aortic PP (mmHg) & $33.92 \pm 6.19$ & $33.55 \pm 5.85$ & $33.82 \pm 6.17$ & $33.40 \pm 5.81$ & $33.86 \pm 6.20$ & $33.45 \pm 5.80$ & $33.79 \pm 6.18$ & $33.47 \pm 5.75$ \\
\hline Aortic HR (bpm) & $58.08 \pm 8.33$ & $59.39 \pm 8.22$ & $58.35 \pm 8.23$ & $59.71 \pm 8.15$ & $58.37 \pm 8.29$ & $59.86 \pm 8.27$ & $58.41 \pm 8.19$ & $59.81 \pm 8.08$ \\
\hline $\mathrm{AP}(\mathrm{mmHg})$ & $5.41 \pm 4.89$ & $5.76 \pm 5.05$ & $5.48 \pm 4.89$ & $6.01 \pm 5.42$ & $5.41 \pm 4.86$ & $5.96 \pm 5.37$ & $5.42 \pm 4.90$ & $6.00 \pm 5.43$ \\
\hline Alx & $14.83 \pm 12.25$ & $15.83 \pm 13.18$ & $15.06 \pm 12.25$ & $16.74 \pm 12.96$ & $15.12 \pm 12.01$ & $16.61 \pm 12.86$ & $15.18 \pm 12.10$ & $16.67 \pm 13.04$ \\
\hline Alx@75 & $6.90 \pm 13.59$ & $8.36 \pm 14.77$ & $7.25 \pm 13.58$ & $9.42 \pm 14.50$ & $7.04 \pm 13.57$ & $9.36 \pm 14.45$ & $7.11 \pm 13.64$ & $9.39 \pm 14.53$ \\
\hline
\end{tabular}

Table 6. Reactive hyperaemia index (RHI) scores across time and treatment groups. ${ }^{1}$

\begin{tabular}{lllll} 
& Placebo $(\mathrm{n}=\mathbf{2 1})$ & Bifidobacterium lactis BL04 $(\mathrm{n}=\mathbf{2 4})$ & B. lactis +PreforPro $(\mathrm{n}=19)$ & Bacillus subtilis $\mathrm{DE}$ 111 $(\mathrm{n}=\mathbf{2 2})$ \\
$\mathrm{RHI}(\mathrm{Pre})$ & $2.14 \pm 0.64$ & $2.42 \pm 0.83$ & $2.27 \pm 0.54$ & $1.97 \pm 0.48$ \\
$\mathrm{RHI}(\mathrm{Post})$ & $2.22 \pm 0.47$ & $2.52 \pm 0.71$ & $2.10 \pm 0.54$ & $2.15 \pm 0.64$ \\
$\Delta \mathrm{RHI}$ & $0.078 \pm 0.69$ & $0.097 \pm 0.81$ & $-0.17 \pm 0.76$ & $0.18 \pm 0.44$ \\
$P$-value & 0.61 & 0.56 & 0.35 & $0.05^{\#}$ \\
\hline
\end{tabular}

${ }^{1}$ Data represents mean \pm standard deviation. No values were found to be statistically different. $\#$ indicates values that trended toward significance $(P=0.05-0.08)$.

\section{Plasma lipids}

Similar to the above section we conducted a one-way ANOVA to compare group baseline values, which revealed no significant differences in baseline variability across groups (Table 7). Significant improvements in TC $(P=0.04$; CI: $-13.40,-0.19)$, and non-HDL-c $(P=0.01 ; \mathrm{CI}:-12.43,-2.07)$ were observed in participants supplemented $B$. subtilis (Figure $2 \mathrm{~B}, \mathrm{C}$ ). In addition, there was a trending reduction in LDL-c from baseline to follow-up (Figure 2D; $P=0.06$; CI: $-12.29,2.87)$. However, there were no significant differences from baseline to post-treatment in any of the blood lipid parameters for the Placebo or either group consuming $B$. lactis containing probiotic. Although there were no statistically significant differences in the baseline adjusted change when comparing the treatments to the placebo, B. subtilis treatment had a very large (values $>1.2$ ) effect on TC (Cohen's $d=3.35$ ), n-HDL-c (Cohen's $d=2.35$ ), and LDL-c (Cohen's $d=4.051)$.

\section{Discussion and conclusions}

The purpose of this pilot intervention study was to determine whether various cardiovascular parameters in a human population could be altered by pro/prebiotic supplementation. A number of studies have explored B. subtilis supplementation for its tolerability in humans as well as and its cardiovascular effects in animal models (Ghoneim et al., 2016; Hanifi et al., 2015; Seo et al., 2010). In the present study, we observed the effects of $B$. subtilis DE111 on endothelial function and plasma lipids over the course of a 4-week intervention. To our knowledge, this is the first randomised controlled trial to investigate the effects of $B$. subtilis supplementation on these parameters in a human population. In addition, we further explored the impact of an E. coli-targeting bacteriophage cocktail (Febvre et al., 2019) and B. lactis on cardiovascular parameters (Bernini et al., 2016; Matsumoto et al., 2019).

We observed modest improvements in endothelial function and significant changes in several plasma lipids in healthy adults supplemented with $B$. subtilis DE111. Previously, Hitosugi et al. showed improvements in visual analog scale scores for shoulder stiffness, low back pain, and coldness in the extremities as proxy measures of blood flow after female participants consumed a fermented soy supplement containing proteases from $B$. subtilis (Hitosugi et al., 2015). While these results may be indicative of improved endothelial function, the investigators did not test consumption of the bacterial spores or directly 

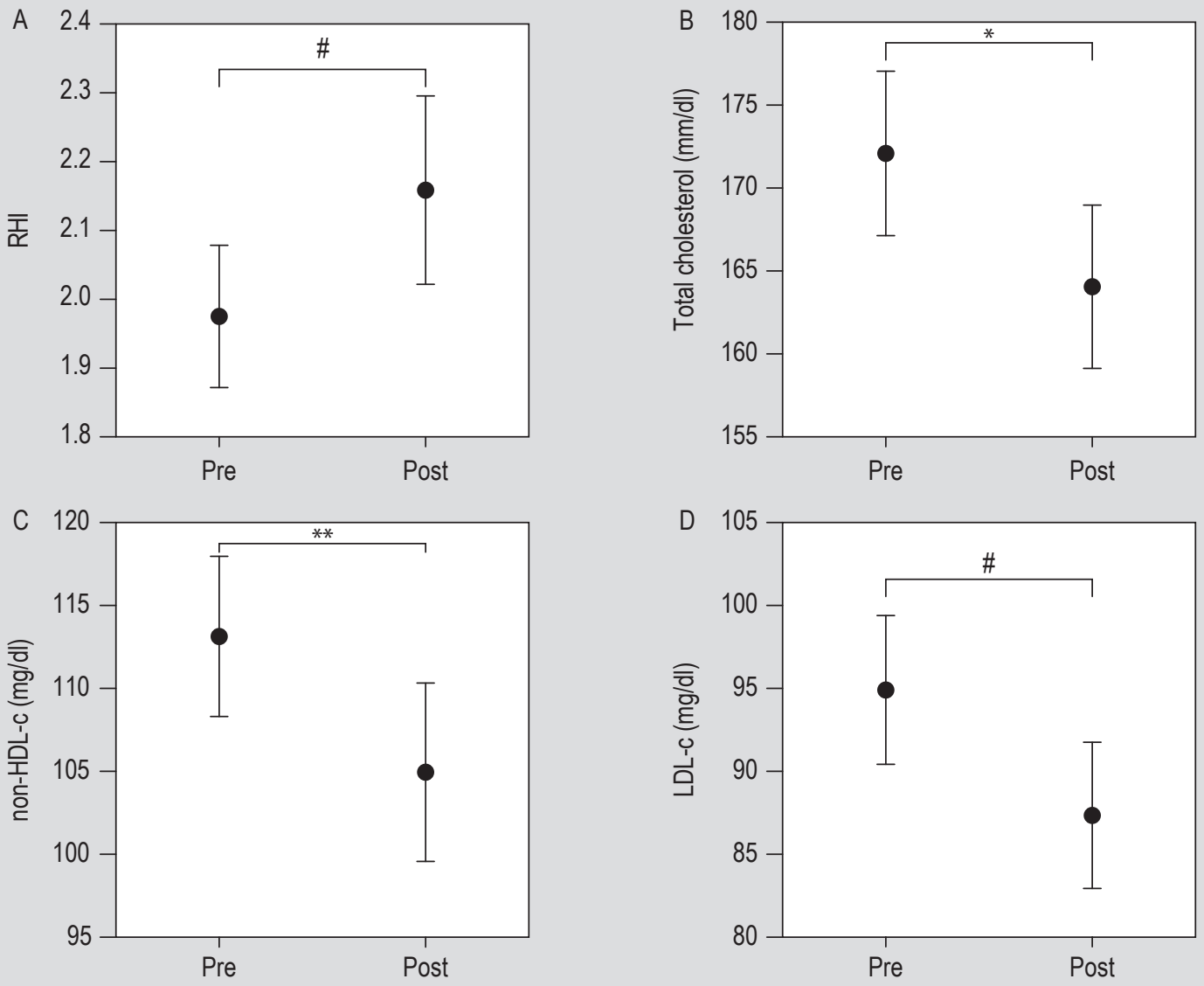

Figure 2. Mean values for reactive hyperaemia index (RHI), total cholesterol (TC), non-high-density lipoprotein-cholesterol (nonHDL-c) and low-density lipoprotein-cholesterol (LDL-c) at baseline and after 4-weeks of Bacillus subtilis DE111 treatment. Error bars represent the standard error of the mean, ${ }^{*} P<0.05$; ${ }^{*} P<0.001$, ${ }^{\#} P=0.05-0.08$.

Table 7. Plasma lipid profiles across time and treatment groups.

\begin{tabular}{|c|c|c|c|c|c|c|c|c|}
\hline & \multicolumn{2}{|c|}{ Placebo (n=19) } & \multicolumn{2}{|c|}{$\begin{array}{l}\text { Bifidobacterium lactis } \\
\text { BL04 }(n=23)\end{array}$} & \multicolumn{2}{|c|}{$\begin{array}{l}\text { B. lactis +PreforPro } \\
(n=19)\end{array}$} & \multicolumn{2}{|c|}{$\begin{array}{l}\text { Bacillus subtilis } \\
\text { DE111 }(n=20)\end{array}$} \\
\hline & Pre & Post & Pre & Post & Pre & Post & Pre & Post \\
\hline TC (mg/dl) & $177.9 \pm 28.97$ & $173.1 \pm 29.50$ & $180.4 \pm 43.75$ & $179.0 \pm 40.12$ & $184.9 \pm 31.55$ & $180.7 \pm 38.46$ & $172.1 \pm 23.26^{*}$ & $164.1 \pm 22.02^{*}$ \\
\hline HDL-c (mg/dl) & $65.70 \pm 13.60$ & $62.79 \pm 15.10$ & $64.13 \pm 15.10$ & $63.21 \pm 15.50$ & $62.57 \pm 13.72$ & $60.42 \pm 13.06$ & $59.00 \pm 15.54$ & $59.30 \pm 18.40$ \\
\hline Triglycerides (mg/dl) & $91.50 \pm 50.94$ & $89.42 \pm 57.42$ & $79.30 \pm 30.87$ & $77.58 \pm 33.16$ & $92.76 \pm 55.58$ & $83.47 \pm 28.60$ & $91.05 \pm 65.05$ & $88.65 \pm 46.55$ \\
\hline nHDL-c (mg/dl) & $112.2 \pm 29.03$ & $110.2 \pm 25.34$ & $116.3 \pm 36.24$ & $115.5 \pm 30.80$ & $122.3 \pm 31.98$ & $120.1 \pm 37.23$ & $113.1 \pm 22.66^{\star *}$ & $105.0 \pm 24.07^{\star \star}$ \\
\hline $\mathrm{TC} / \mathrm{H}$ & $2.79 \pm 0.58$ & $2.84 \pm 0.54$ & $2.88 \pm 0.72$ & $2.90 \pm 0.68$ & $3.08 \pm 0.85$ & $3.08 \pm 0.84$ & $3.07 \pm 0.78$ & $3.00 \pm 0.94$ \\
\hline LDL-c (mg/dl) & $94.0 \pm 28.29$ & $92.16 \pm 26.81$ & $100.4 \pm 32.08$ & $100.0 \pm 27.20$ & $103.8 \pm 27.73$ & $103.3 \pm 36.03$ & $94.91 \pm 21.04$ & $87.35 \pm 19.70^{\#}$ \\
\hline VLDL-c (mg/dl) & $18.35 \pm 10.24$ & $17.84 \pm 11.49$ & $15.91 \pm 6.19$ & $15.38 \pm 6.68$ & $18.52 \pm 11.21$ & $16.74 \pm 5.83$ & $18.23 \pm 12.92$ & $17.70 \pm 9.22$ \\
\hline
\end{tabular}

${ }^{1}$ Data represents mean $\pm S D$. Values that are statistically different are indicated with an asterisk ${ }^{*} P<0.05,{ }^{* *} P<0.01,{ }^{\#} P=0.05-0.08$.

${ }^{2} \mathrm{TC}=$ total cholesterol; HDL-c = high-density lipoprotein-cholesterol; $\mathrm{nHDL}-\mathrm{c}=$ non-high-density lipoprotein-cholesterol; LDL-c = low-density lipoproteincholesterol; VLDL-c = very low-density lipoprotein-cholesterol. 
measure endothelial function. Considering the relatively short duration of the current intervention (4 weeks), and the healthy status of the study participants, further exploration of the potential cardiovascular benefits of B. subtilis is warranted in clinical populations. The current study provides a good basis for determining an appropriate sample size for adequately powering future clinical interventions.

Bacillus subtilis supplementation also resulted in a cholesterol lowering effect on TC, LDL, and non-HDL cholesterol, although HDL-c was unaffected. These data are consistent with observations from previously conducted studies in animal models (Ghoneim et al., 2016; Seo et al., 2010; Zouari et al., 2015). Ghoneim et al. reported improvements in total cholesterol, LDL-c, VLDL-c, HDL-c, and triglycerides in Streptozotocin-induced diabetic rats after administration of the exopolysaccharide from a wild collected strain of B. subtilis (Ghoneim et al., 2016). Likewise, cholesterol lowering effects were noted in ApoE knockout mice fed an atherogenic diet supplemented with soymilk fermented by B. subtilis (Seo et al., 2010). Although the changes we observed in these parameters were only statistically significant within a treatment group (pre- to post-intervention), the effect size (measured as Cohen's $d$ ) relative to the placebo were very large, suggesting these changes may be clinically relevant. Reducing cholesterol concentrations has profound effects on lowering risk for developing coronary heart disease (CHD); lowering cholesterol levels by as little as $1 \%$ can reduce CHD risk by 2-3\% (Kumar et al., 2012). While there are no clear mechanisms to explain the cholesterol lowering effects of $B$. subtilis, it is conceivable that it may be due to the effect short chain fatty acids (SCFA) have on cholesterol metabolism. As previously mentioned, insoluble fibre is fermented in the small intestine by a variety of bacterial species, producing SCFA. While B. subtilis is known to stimulate the production of lactic acid by enhancing the growth of Lactobacillus spp., lactic acid can also be converted to SCFA through cross feeding (Schauf et al., 2019). SCFA have been demonstrated to inhibit the synthesis of hepatic cholesterol (VLDL-c), leading further to a reduction in LDL-c and non-HDL-c. SCFA may also inhibit VLDL-c synthesis through the inhibition of pyruvate dehydrogenase via the formation of acetyl CoA, affecting hepatic ketogenesis through the delivery of ketone precursors, or altering the oxidation-reduction state (Fechner et al., 2014). In addition, it is known that the introduction of new bacterial species can enhance the growth of other species, such as SCFA producing bacteria. Remarkably, it has also been reported that humans with atherosclerosis display reduced levels of butyrate-producing genera Butyrivibrio and Roseburia (Karlsson et al., 2012). B. subtilis supplementation may have an indirect effect on enhancing the generation of SCFA leading to this cholesterol lowering effect. It would be worthwhile to examine this parameter in future studies.
We did not observe any significant effects on endothelial function or plasma lipids in individuals supplemented with B. lactis, with or without bacteriophages, findings that are not concordant with observations from several other published human trials (Bernini et al., 2016; Matsumoto et al., 2019). For example, body weight, TC and LDL-c were significantly reduced in participants supplemented with B. lactis fermented milk for 45 days (Bernini et al., 2016). Matsumoto et al. saw improvements in RHI in normal weight, healthy individuals after 12 weeks consumption of a B. lactis + arginine containing yogurt (Matsumoto et al., 2019). However, several key differences exist between these studies and the current study that may explain these discrepancies. First, both studies provided the supplementation for a longer period of time, suggesting that our study duration may have been insufficient to observe any beneficial effects on cardiovascular parameters. In addition, both studies used fermented milk products as a delivery vehicle for the probiotic. Milk contains calcium, vitamin D (supplemented), conjugated linoleic acid, bioactive peptides and numerous other components that may independently influence lipid metabolism and cardiovascular health (Mozaffarian and Wu, 2018; Rice, 2014). Furthermore, Bernini et al. reported improvements in a population with metabolic syndrome, rather than a healthy population, suggesting that individuals with dyslipidaemia at baseline may have a greater response to Bifidobacterium. Finally, the Matsumoto study also supplemented with arginine, a precursor of the vasodilatory molecule nitric oxide, which may have contributed to the vascular improvements reported in their study.

There are several noteworthy strengths and limitations of the current study. The randomised, double-blind, placebocontrolled study design is the gold standard for a clinical trial of this nature. While compliance was generally good based on returned unused capsules, there were no additional objective measures used to determine if participants were compliant with the daily treatment protocol. In addition, although we requested that participants complete two 24-hour dietary recalls at baseline and trial completion, several participants did not report at all and some only provided baseline diet data or a single day's food recall and there was no assessment of physical activity. Despite these shortcomings, consistency in baseline and posttreatment body weight, along with the limited reliable diet data obtained, indicate that participants largely maintained typical consumption and physical activity patterns.

In summary, CVD is a world-wide epidemic and as the global population ages, it is likely the prevalence of CVD will continue to increase (Johnson et al., 2019). Hence it is imperative to uncover cost effective, therapeutic approaches for the prevention of CVD. Bacillus subtilis DE111 supplementation over the course of 4 weeks resulted in a modest increase in mean RHI score, a measure of endothelial function, as well as significant improvements 
in TC and non-HDL-c levels. These improvements show promise to future research and the health benefits of $B$. subtilis on CVD risk. While we did not observe an effect of $B$. lactis supplementation with or without bacteriophages, further research is necessary to examine its effects in a clinical population. In total, the results from this study warrant further research in probiotics and bacteriophage supplementation in humans as a novel approach reducing the risk for CVD.

\section{References}

Battson, M.L., Lee, D.M., Jarrell, D.K., Hou, S., Ecton, K.E., Weir, T.L. and Gentile, C.L., 2018a. Suppression of gut dysbiosis reverses Western diet-induced vascular dysfunction. American Journal of Physiology - Endocrinology and Metabolism 314: E468-E477. https://doi.org/10.1152/ajpendo.00187.2017

Battson, M.L., Lee, D.M., Weir, T.L. and Gentile, C.L., 2018b. The gut microbiota as a novel regulator of cardiovascular function and disease. Journal of Nutritional Biochemistry 56: 1-15. https://doi. org/10.1016/j.jnutbio.2017.12.010

Bernini, L.J., Simão, A.N.C., Alfieri, D.F., Lozovoy, M.A.B., Mari, N.L., De Souza, C.H.B., Dichi, I. and Costa, G.N., 2016. Beneficial effects of Bifidobacterium lactis on lipid profile and cytokines in patients with metabolic syndrome: a randomized trial. Effects of probiotics on metabolic syndrome. Nutrition 32: 716-719. https:// doi.org/10.1016/j.nut.2015.11.001

Cani, P.D., Bibiloni, R., Knauf, C., Waget, A., Neyrinck, A.M., Delzenne, N.M. and Burcelin, R., 2008. Changes in gut microbiota control metabolic endotoxemia-induced inflammation in high-fat dietinduced obesity and diabetes in mice. Diabetes 57: 1470-1481. https://doi.org/10.2337/db07-1403

Cohen, J., 2013. Statistical power analysis for the behavioral sciences. Taylor and Francis, London, UK.

Febvre, H., Rao, S., Gindin, M., Goodwin, N., Finer, E., Vivanco, J., Lu, S., Manter, D., Wallace, T. and Weir, T., 2019. PHAGE study: effects of supplemental bacteriophage intake on inflammation and gut microbiota in healthy adults. Nutrients 11: 666. https://doi. org/10.3390/nu11030666

Fechner, A., Kiehntopf, M. and Jahreis, G., 2014. The formation of short-chain fatty acids is positively associated with the blood lipid-lowering effect of lupin kernel fiber in moderately hypercholesterolemic adults. Journal of Nutrition 144: 599-607. https://doi.org/10.3945/jn.113.186858

Ghoneim, M.A.M., Hassan, A.I., Mahmoud, M.G. and Asker, M.S., 2016. Effect of polysaccharide from Bacillus subtilis sp. on cardiovascular diseases and atherogenic indices in diabetic rats. BMC Complementary and Alternative Medicine 16: 112. https:// doi.org/10.1186/s12906-016-1093-1

Gibson, G.R., Hutkins, R., Sanders, M.E., Prescott, S.L., Reimer, R.A., Salminen, S.J., Scott, K., Stanton, C., Swanson, K.S., Cani, P.D., Verbeke, K. and Reid, G., 2017. Expert consensus document: The International Scientific Association for Probiotics and Prebiotics (ISAPP) consensus statement on the definition and scope of prebiotics. Nature Reviews Gastroenterology and Hepatology 14: 491-502. https://doi.org/10.1038/nrgastro.2017.75
Hanifi, A., Culpepper, T., Mai, V., Anand, A., Ford, A.L., Ukhanova, M., Christman, M., Tompkins, T.A. and Dahl, W.J., 2015. Evaluation of Bacillus subtilis R0179 on gastrointestinal viability and general wellness: a randomised, double-blind, placebo-controlled trial in healthy adults. Beneficial Microbes 6: 19-27. https://doi.org/10.3920/ BM2014.0031

Hill, C., Guarner, F., Reid, G., Gibson, G.R., Merenstein, D.J., Pot, B., Morelli, L., Canani, R.B., Flint, H.J., Salminen, S., Calder, P.C. and Sanders, M.E. 2014. The International Scientific Association for Probiotics and Prebiotics consensus statement on the scope and appropriate use of the term probiotic. Nature Reviews Gastroenterology and Hepatology 11: 506-514. https://doi. org/10.1038/nrgastro.2014.66

Hitosugi, M., Hamada, K. and Misaka, K. 2015. Effects of Bacillus subtilis var. Natto products on symptoms caused by blood flow disturbance in female patients with lifestyle diseases. International Journal of General Medicine 2015: 41-46. https://doi.org/10.2147/ IJGM.S76588

Johnson, S.A., Litwin, N.S. and Seals, D.R., 2019. Age-related vascular dysfunction: what registered dietitian nutritionists need to know. Practice Applications Professional Practice| 119: 1790-1796. https:// doi.org/10.1016/j.jand.2019.03.016

Karlsson, F.H., Fåk, F., Nookaew, I., Tremaroli, V., Fagerberg, B., Petranovic, D., Bäckhed, F. and Nielsen, J., 2012. Symptomatic atherosclerosis is associated with an altered gut metagenome. Nature Communications 3: 1245. https://doi.org/10.1038/ncomms2266

Kelly, R.P., Millasseau, S.C., Ritter, J.M. and Chowienczyk, P.J., 2001. Vasoactive drugs influence aortic augmentation index independently of pulse-wave velocity in healthy men. Hypertension 37: 1429-1433. https://doi.org/10.1161/01.HYP.37.6.1429

Khalesi, S., Sun, J., Buys, N. and Jayasinghe, R., 2014. Effect of probiotics on blood pressure: a systematic review and meta-analysis of randomized, controlled trials. Hypertension 64: 897-903. https:// doi.org/10.1161/hypertensionaha.114.03469

Koren, O., Spor, A., Felin, J., Fak, F., Stombaugh, J., Tremaroli, V., Behre, C.J., Knight, R., Fagerberg, B., Ley, R.E. and Backhed, F., 2011. Human oral, gut, and plaque microbiota in patients with atherosclerosis. Proceedings of the National Academy of Sciences of the USA 108, Suppl. 1: 4592-4598. https://doi.org/10.1073/ pnas.1011383107

Kumar, M., Nagpal, R., Kumar, R., Hemalatha, R., Verma, V., Kumar, A., Chakraborty, C., Singh, B., Marotta, F., Jain, S. and Yadav, H. 2012. Cholesterol-lowering probiotics as potential biotherapeutics for metabolic diseases. Experimental Diabetes Research 2012: 902917. https://doi.org/10.1155/2012/902917

Litwin, N.S., Van Ark, H.J., Hartley, S.C., Michell, K.A., Vazquez, A.R., Fischer, E.K., Melby, C.L., Weir, T.L., Wei, Y., Rao, S., Hildreth, K.L., Seals, D.R., Pagliassotti, M.J. and Johnson, S.A., 2019. Impact of red beetroot juice on vascular endothelial function and cardiometabolic responses to a high-fat meal in middle-aged/older adults with overweight and obesity: a randomized, double-blind, placebocontrolled, crossover trial. Current Developments in Nutrition 3: 113. https://doi.org/10.1093/cdn/nzz113 
Malik, M., Suboc, T.M., Tyagi, S., Salzman, N., Wang, J., Ying, R., Tanner, M.J., Kakarla, M., Baker, J.E. and Widlansky, M.E., 2018. Lactobacillus plantarum 299v supplementation improves vascular endothelial function and reduces inflammatory biomarkers in men with stable cronary artery disease. Circulation Research 123: 10911102. https://doi.org/10.1161/CIRCRESAHA.118.313565

Matsumoto, M., Kitada, Y. and Naito, Y., 2019. Endothelial function is improved by inducing microbial polyamine production in the gut: a randomized placebo-controlled trial. Nutrients 11: 1188. https:// doi.org/10.3390/nu11051188

Mozaffarian, D. and Wu, J.H.Y., 2018. Flavonoids, dairy foods, and cardiovascular and metabolic health: a review of emerging biologic pathways. Circulation Research 122: 369-384. https://doi. org/10.1161/circresaha.117.309008

Rice, B.H., 2014. Dairy and cardiovascular disease: a review of recent observational research. Current Nutrition Reports 3: 130-138. https://doi.org/10.1007/s13668-014-0076-4.

Sawilowsky, S.S., 2009. New effect size rules of thumb. Journal of Modern Applied Statistical Methods 8: 597-599. https://doi. org/10.22237/jmasm/1257035100

Schauf, S., Nakamura, N. and Castrillo, C., 2019. Effect of Calsporin ${ }^{\oplus}$ (Bacillus subtilis C-3102) addition to the diet on faecal quality and nutrient digestibility in healthy adult dogs. Journal of Applied Animal Nutrition 7: e3. https://doi.org/10.1017/jan.2019.2

Seo, K.-C., Kim, M.-J., Hong, S.-H., Cha, S.-Y., Noh, J.-S., Kim, M.-J. and Song, Y.-O., 2010. The hypocholesterolemic effects of soymilk fermented with Bacillus subtilis compared to soymilk with Cheonggukjang powder in apolipoprotein e knockout mice. Preventive Nutrition and Food Science 15: 83-87. https://doi. org/10.3746/jfn.2010.15.2.083
Szulińska, M., Łoniewski, I., Skrypnik, K., Sobieska, M., Korybalska, K., Suliburska, J. and Bogdański, P., 2018. Multispecies probiotic supplementation favorably affects vascular function and reduces arterial stiffness in obese postmenopausal women - a 12-week placebo-controlled and randomized clinical study. Nutrients 10 : 1672. https://doi.org/10.3390/nu10111672

Xie, N., Cui, Y., Yin, Y.-N., Zhao, X., Yang, J.-W., Wang, Z.-G., Fu, N., Tang, Y., Wang, X.-H., Liu, X.-W., Wang, C.-L. and Lu, F.-G. 2011. Effects of two Lactobacillus strains on lipid metabolism and intestinal microflora in rats fed a high-cholesterol diet. BMC Complementary and Alternative Medicine 11: 53. https://doi. org/10.1186/1472-6882-11-53

Yelland, L.N., Sullivan, T.R., Voysey, M., Lee, K.J., Cook, J.A. and Forbes, A.B., 2015. Applying the intention-to-treat principle in practice: guidance on handling randomisation errors. Clinical Trials 12: 418-423. https://doi.org/10.1177/1740774515588097

Ziganshina, E.E., Sharifullina, D.M., Lozhkin, A.P., Khayrullin, R.N., Ignatyev, I.M. and Ziganshin, A.M. 2016. Bacterial communities associated with atherosclerotic plaques from Russian individuals with atherosclerosis. PLoS ONE 11: e0164836. https://doi. org/10.1371/journal.pone.0164836

Zouari, R., Ben Abdallah-Kolsi, R., Hamden, K., Feki, A.E., Chaabouni, K., Makni-Ayadi, F., Sallemi, F., Ellouze-Chaabouni, S. and GhribiAydi, D., 2015. Assessment of the antidiabetic and antilipidemic properties of Bacillus subtilis spb1 biosurfactant in alloxan-induced diabetic rats: antidiabetic and antilipidemic properties of Bacillus subtilis SPB1 biosurfactant. Biopolymers 104: 764-774. https://doi. org/10.1002/bip.22705 